Document downloaded from:

http://hdl.handle.net/10251/56142

This paper must be cited as:

Moragues Escrivá, J.; Vergara Domínguez, L.; Gosálbez Castillo, J. (2011). Generalized matched subspace filter for dependent noise based on ICA. IEEE Transactions on Signal Processing. 59(7):3430-3434. doi:10.1109/TSP.2011.2141668.

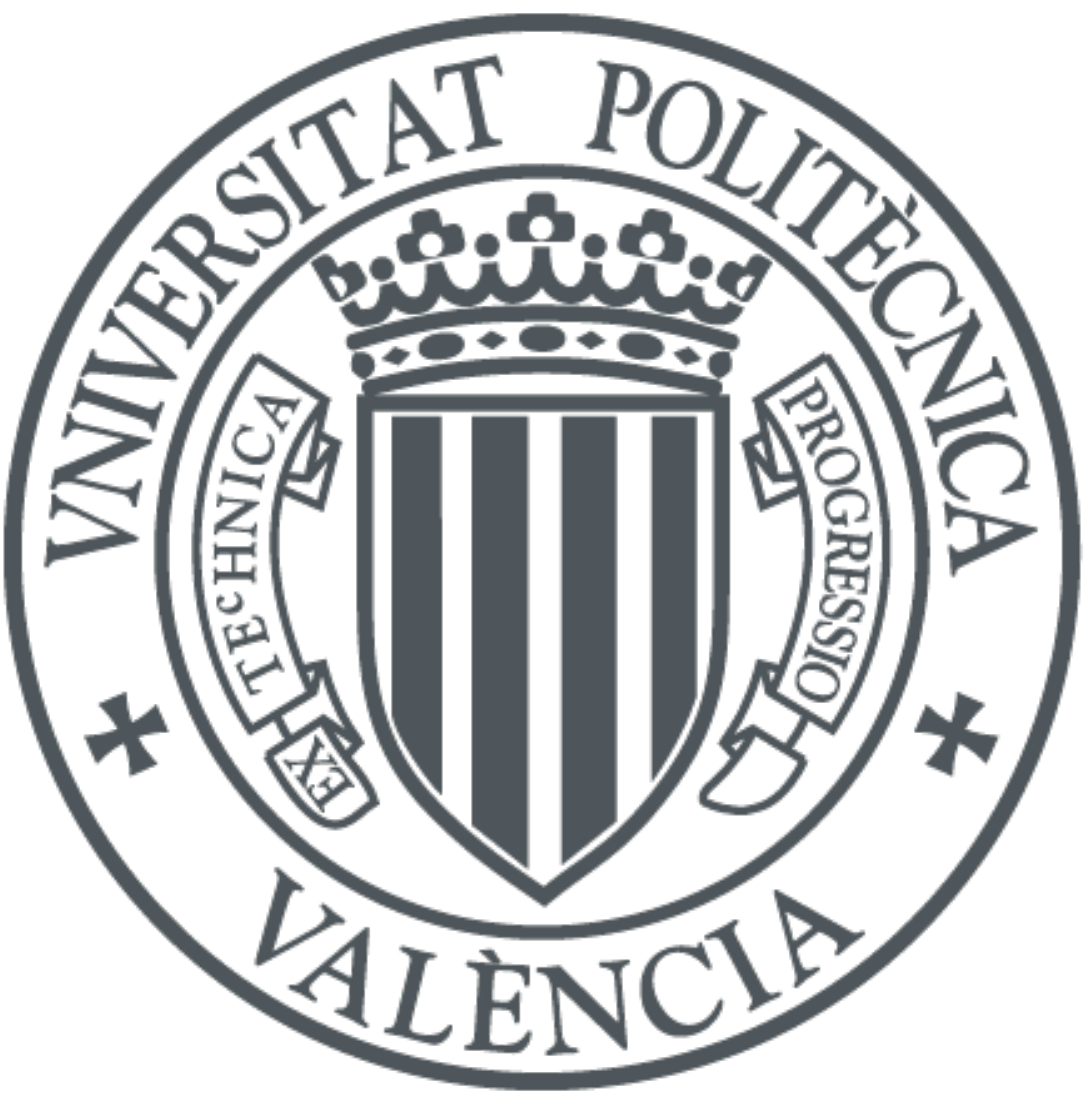

The final publication is available at

http://dx.doi.org/10.1109/TSP.2011.2141668

Copyright Institute of Electrical and Electronics Engineers (IEEE)

Additional Information 


\title{
Generalized matched subspace filter for non-independent noise based on ICA
}

\author{
J. Moragues*, L. Vergara, and J. Gosálbez
}

\begin{abstract}
We propose a generalization of the matched subspace filters for the detection of unknown signals in a background of non-Gaussian and non-independent noise. The generalization is based on a modification of the Rao test by including a linear transformation derived from Independent Component Analysis (ICA). Receiver Operating Characteristic (ROC) curves computed for simulated examples illustrate the significant improvement achieved with the generalized solution.
\end{abstract}

Index Terms-Rao test, matched subspace filter, non-Gaussian noise, ICA.

\section{INTRODUCTION}

Matched subspace filter (MSF) is known to be an uniformly most powerful (UMP) detector for the detection of a subspace signal in a background of uncorrelated Gaussian noise [1]. Furthermore, it is the optimum solution for the following hypothesis problem:

$$
\begin{array}{ll}
H_{0}: \mathbf{y}=\mathbf{w} & \mathbf{w}: N\left(0, \sigma_{\mathbf{w}}^{2} \mathbf{I}\right) \\
H_{1}: \mathbf{y}=\mu \mathbf{s}+\mathbf{w} & \mathbf{s}: \mathbf{H} \boldsymbol{\theta}
\end{array}
$$

where $\mathbf{y}$ is the observation vector in each hypothesis (dimension $N$ ), $\mathbf{w}$ is the zero-mean multivariate Gaussian noise vector and $\mu \mathbf{s}$ is the signal vector. It is assumed that $\mathbf{s}$ lies in a subspace spanned by the $p<N$ columns of a known matrix $\mathbf{H}(N \times p)$ and that the model parameters $\mu$ and $\boldsymbol{\theta}$ are unknown. The MSF is based on measuring the observation energy included in the signal subspace by implementing the following hypothesis test (real case is assumed):

$$
\frac{\mathbf{y}^{T} \mathbf{P y}}{\sigma_{\mathbf{w}}^{2}} \underset{H_{0}}{\stackrel{H_{1}}{<}} \lambda
$$

where $\mathbf{P}$ is the projection matrix onto the signal subspace and it is given by $\mathbf{P}=\mathbf{H}\left(\mathbf{H}^{T} \mathbf{H}\right)^{-1} \mathbf{H}^{T}$. The statistic $\frac{\mathbf{y}^{T} \mathbf{P y}}{\sigma_{\mathbf{w}}^{2}}$ is chi-squared distributed with $p$ degrees of freedom $\left(\chi_{p}^{2}\right)$, so that the threshold value $\lambda$ may be obtained for a required probability of false alarm (PFA).

Optimality of the MSF is kept even in the presence of subspace interferences [2]. Also in [3], similar detector solutions are proposed in the presence of interference for specific types of independent nonGaussian noise (called generalized Gaussian distributions). Unfortunately, there is not any general UMP solution for the subspace signal detection problem when the noise is non-Gaussian and nonindependent. Hence, suboptimal detectors are to be devised. A generalized likelihood ratio test (GLRT) could be implemented, but it requires Maximum Likelihood (ML) estimates of the unknown parameters (in our case $\mu$ and $\boldsymbol{\theta}$ ), which is not a very practical option. Other well-known suboptimal alternatives exist, like the Wald and Rao tests [4]. The Wald test, although simpler to implement than the GLRT, also requires estimates of the involved parameters under $H_{1}$. However, the Rao test does not have such a requirement as in its most

Copyright (c) 2011 IEEE. Personal use of this material is permitted. However, permission to use this material for any other purposes must be obtained from the IEEE by sending a request to pubs-permissions@ieee.org.

The authors are with the "Instituto de Telecomunicaciones y Aplicaciones Multimedia (iTEAM)" in "Universidad Politécnica de Valencia", 46022 Valencia, SPAIN (e-mail: jormoes@upvnet.upv.es; Tel:+34963877308; Fax: $+34963877919)$ general form only needs ML estimates of the nuisance parameters under $H_{0}$. Notice that in the considered problem, the vector of parameters is given by $\boldsymbol{\theta}_{r}=\mathbf{0}$ (under $H_{0}$ ) and $\boldsymbol{\theta}_{r}=\left[\mu, \boldsymbol{\theta}^{T}\right]^{T}$ (under $H_{1}$ ), i.e., no nuisance parameters appear and Rao test becomes an attractive alternative to deal with the non-Gaussian noise case. It is relevant to mention that in any case GLRT, Wald and Rao test are asymptotically equivalent. Moreover it has been recently shown that, for a finite number of observations, the three tests are coincident or statistically equivalent in a number of typical detection problems [5] [6]. In particular, it is demonstrated in [6] that coincidence exists in detection problems without nuisance parameters when the observation probability density function (PDF) belongs to the exponential family, thus covering a broad range of practical cases.

In consequence, we will focus our attention on the Rao test. It is rather simple to adapt the general form of the Rao test to the signal subspace detection problem assuming that the components of the noise vector $\mathbf{w}$ are independent and identically distributed (i.i.d.) random variables. This has been done in [4], arriving to the hypothesis test

$$
\frac{g(\mathbf{y})^{T} \mathbf{P} g(\mathbf{y})}{P_{g(w)}} \underset{H_{0}}{\stackrel{H_{1}}{\gtrless}} \lambda
$$

where function $g($.$) is independently applied to each element of$ vector $\mathbf{y}$ in the form $g(\mathbf{y})=\left[g\left(y_{0}\right) g\left(y_{1}\right) \ldots g\left(y_{N-1}\right)\right]^{T}$. If $p(w)$ is the noise PDF, we can define

$$
g(w)=-\frac{\frac{d p(w)}{d w}}{p(w)} \text { and } P_{g(w)}=\int_{-\infty}^{\infty} \frac{\left[\frac{d p(w)}{d w}\right]^{2}}{p(w)} d w
$$

Notice that $P_{g(w)}$ is the preprocessed noise mean-power and can be expressed as follows:

$$
P_{g(w)}=\int_{-\infty}^{\infty} g^{2}(w) p(w) d w=E\left[g^{2}(w)\right],
$$

where $E[$.$] means statistical expectation. Therefore, (3) is an exten-$ sion of the MSF in the sense that some nonlinear transformation $g($. is applied to the original observation vector prior to the computation of the normalized subspace energy. Note that for the Gaussian case $g(w)=w$, therefore $g(\mathbf{y})=\mathbf{y}$ and $P_{g(w)}=P_{w}=\sigma_{w}^{2}$, i.e. (2) and (3) are equivalent.

In addition to the already mentioned equivalences with the GLRT and the Wald test, the Rao test has the interesting property that the involved statistic $g(\mathbf{y})^{T} \mathbf{P} g(\mathbf{y}) / P_{g(w)}$ is $\chi_{p}^{2}$ as in (2), hence we can easily compute the value $\lambda$ for a required PFA. In the following, we will denote as MSF the extension defined in (3) suitable for independent and non-Gaussian noise.

In this work, we propose a further generalization of the MSF, called generalized MSF (GMSF), in order to consider the most general case of non-Gaussian and non-independent noise. It is based on the use of Independent Component Analysis (ICA), a generalization of Principal Component Analysis (PCA), [7] [8], which implements a matrix linear transformation to make the components of the transformed observation vector as independent as possible. The same idea was exploited in [9] to derive energy detectors in the presence of nonGaussian noise. Actually, ICA could be resorted to implement a 
linear pre-processing step in any detection problem involving nonGaussianity and statistical dependence among the observed vector components. However, each detector requires a particular attention due to the specific implementation of the non-linear transformation $g($.$) . In [9], an "ad hoc" non-linear function is proposed to make as$ Gaussian as possible the linearly transformed observation vector, but the GLRT condition of the extended energy detector used is not generally demonstrated. Nevertheless, this is not the case in the detection problem considered in this work since the non-linear transformation defined in (4) is inherent to the Rao test, thus preserving its properties.

The paper is organized as follows. In Section II, the GMSF is defined and the computations required to implement it are given. In Section III, the experimental setup and the simulation results are included in order to verify the improvement of the GMSF with respect to the MSF when the noise is neither Gaussian nor independent. Finally, the conclusions of our work are mentioned in Section IV.

\section{Generalized MATCHED SUBSPACE FILTER}

Test (3) assumes that the components of $\mathbf{w}$ are i.i.d. When this is not the case, we could transform the non-independent observation vector into a new one having independent components. This can be done by means of ICA and an appropriate linear transformation. Let us call $\mathbf{U}$ the transformation used to obtain i.i.d. vector noise samples by means of $\mathbf{u}=\mathbf{U w}$. We propose the GMSF in the form

$$
\frac{g(\mathbf{U y})^{T} \mathbf{P}_{U} g(\mathbf{U y})}{P_{g(u)}} \underset{H_{0}}{\stackrel{H_{1}}{<}} \lambda,
$$

where $P_{g(u)}=E\left[g^{2}(u)\right]$, and $\mathbf{P}_{U}=\mathbf{H}_{U}\left(\mathbf{H}_{U}^{T} \mathbf{H}_{U}\right)^{-1} \mathbf{H}_{U}^{T}$ with $\mathbf{H}_{U}=\mathbf{U H}$. Notice that the non-linear transformation appearing in (6) must be defined from the PDF of the linearly transformed noise samples $u$, which will generally have a different (non-Gaussian) PDF of the original noise $w$. Thereby, using (4) and changing $w$ by $u$ we obtain:

$$
g(u)=-\frac{\frac{d p(u)}{d u}}{p(u)} .
$$

Thus, it is guaranteed that equation (6) implements a Rao test in the linearly transformed observation vector $\mathbf{y}_{\mathbf{U}}=\mathbf{U y}$, hence keeping all the mentioned properties of the Rao test such as the $\chi_{p}^{2}$ distribution of the statistic $g(\mathbf{U y})^{T} \mathbf{P}_{U} g(\mathbf{U y}) / P_{g(u)}$ and the asymptotic equivalence with the GLRT and Wald test.

Different criteria have been proposed to estimate the required ICA transformation. In essence, all of them are trying to minimize some appropriate measure of the dependence among the vector components. In particular, $\mathbf{U}$ may be decomposed as follows:

$$
\mathbf{U}=\mathbf{Q R}_{w}^{-\frac{1}{2}}
$$

where $\mathbf{Q}$ is a unitary matrix which performs the required rotation when the observation noise is non-Gaussian and $\mathbf{R}_{w}$ is the noise autocorrelation matrix that can be estimated using the classical unbiased estimator:

$$
\hat{\mathbf{R}}_{w}=\frac{1}{K} \sum_{k=1}^{K} \mathbf{w}_{k} \mathbf{w}_{k}^{T},
$$

where $K$ is the number of available noise vectors used to train the detector. Then, by applying an iterative gradient algorithm, as used in [9], it is possible to estimate the rotation matrix $\mathbf{Q}$ as follows:

$$
\begin{aligned}
& \hat{\mathbf{Q}}_{i+1}^{\prime}=\hat{\mathbf{Q}}_{i}+\beta \sum_{k+1}^{K}\left[\left(\hat{\mathbf{Q}}_{i}^{T}\right)^{-1}-g\left(\hat{\mathbf{Q}}_{i} \mathbf{w}_{p k}\right) \mathbf{w}_{p k}^{T}\right] \\
& \hat{\mathbf{Q}}_{i+1}=\hat{\mathbf{Q}}_{i+1}^{\prime}\left(\hat{\mathbf{Q}}_{i+1}^{\prime T} \hat{\mathbf{Q}}_{i+1}^{\prime}\right)^{-\frac{1}{2}},
\end{aligned}
$$

where $\mathbf{w}_{p k}=\hat{\mathbf{R}}_{\mathbf{w}}^{-\frac{1}{2}} \mathbf{w}_{k}$ are prewhitened training noise vectors and (11) is required to make the estimate of $\mathbf{Q}$ a unitary matrix.

Test (6) and equation (10) require knowledge of the pre-processing function $g($.$) , which depends on the noise PDF p(u)$ as shown in (7). If there is a priori knowledge of $p(u)$, we can directly compute the nonlinear function $g($.$) . For example, if u$ follows a Laplacian PDF:

$$
p(u)=\frac{1}{\sqrt{2 \sigma^{2}}} \exp \left(-\sqrt{\frac{2}{\sigma^{2}}}|u|\right),
$$

where $\sigma^{2}$ is the variance, the pre-processing function can be expressed as:

$$
g(u)=-\frac{\frac{d p(u)}{d u}}{p(u)}=\sqrt{\frac{2}{\sigma^{2}}} \frac{d|u|}{d u} .
$$

But, in general, there will not be any available knowledge about the PDF of $u$, hence we have to use a non-parametric approach. Let us consider the set of samples $\left\{u_{l}\right\}, l=1 \ldots L$ corresponding to realizations of the random variable $u$ whose PDF is to be estimated. The classical non-parametric estimator takes the form [10]:

$$
\hat{p}(u)=a \sum_{l=1}^{L} \exp \left(-\frac{1}{2}\left(\frac{u-u_{l}}{h}\right)^{2}\right)
$$

where $a=\frac{1}{N h \sqrt{2 \pi}}$ is a normalization constant and $h$ is a parameter which controls the degree of smoothing of the estimated PDF (some rule of thumbs are available to fit $h$ ). Let us assume that the quality of the estimate (14) is appropriate to consider $p(u) \cong \hat{p}(u)$, so that we can compute the nonlinear pre-processing function $g($.$) as described$ in (7). We start by computing the derivative of (14) with respect to $u$ as follows

$$
\begin{aligned}
\frac{d p(u)}{d u}= & a \sum_{l=1}^{L} \exp \left(-\frac{1}{2}\left(\frac{u-u_{l}}{h}\right)^{2}\right) \cdot\left(-\frac{u-u_{l}}{h}\right) \frac{1}{h} \\
= & \frac{a}{h^{2}} \sum_{l=1}^{L} u_{l} \cdot \exp \left(-\frac{1}{2}\left(\frac{u-u_{l}}{h}\right)^{2}\right) \\
& -\frac{a}{h^{2}} \sum_{l=1}^{L} u \cdot \exp \left(-\frac{1}{2}\left(\frac{u-u_{l}}{h}\right)^{2}\right)
\end{aligned}
$$

and hence

$$
g(u)=\frac{1}{h^{2}}\left[u-\frac{\sum_{l=1}^{L} u_{l} \cdot \exp \left(-\frac{1}{2}\left(\frac{u-u_{l}}{h}\right)^{2}\right)}{\sum_{l=1}^{L} \exp \left(-\frac{1}{2}\left(\frac{u-u_{l}}{h}\right)^{2}\right)}\right] .
$$

We may identify in (16) a linear and a nonlinear term. This later accounts for the possible non-Gaussianity of the random variable $u$.

\section{EXPERIMENTAL ANALYSIS}

\section{A. Signal model}

In order to evaluate the improvement of the GMSF in comparison to the MSF, different experiments were conducted using (6) and (3) respectively. Thereby, a subspace signal is to be detected in presence of non-Gaussian and non-independent noise. Subspace signals will be considered band-limited formed by the sum of one or more sinusoids as defined in (17). This is a specific subspace signal, but it is of particular interest to study the expected improvements when using 
the GMSF as it will be shown in the experiments performed. Hence, the detection problem is expressed as follows:

$$
\begin{aligned}
& H_{0}: y[n]=w[n] \\
& H_{1}: y[n]=\sum_{m=1}^{M} A_{m} \cos \left(2 \pi m f_{0} n+\phi_{m}\right)+w[n]
\end{aligned}
$$

where $n=0,1, \ldots, N-1$ and $m=1, \ldots, M$ represents the number of sinusoids used. The amplitude $A_{m}$ and phase $\phi_{m}$ are assumed to be unknown, $f_{0}$ is assumed to be known and $w[n]$ are the nonindependent and non-Gaussian noise samples. We can rewrite the data model in the linear model form as $\mathbf{y}=\mathbf{H} \boldsymbol{\theta}+\mathbf{w}$, with the subspace matrix given by:

$$
\begin{aligned}
& \mathbf{H}= {\left[\begin{array}{cc}
1 & 0 \\
\cos \left[\omega_{1}\right] & \sin \left[\omega_{1}\right] \\
\vdots & \vdots \\
\cos \left[\omega_{1}(N-1)\right] & \sin \left[\omega_{1}(N-1)\right]
\end{array}\right) \| } \\
& \ldots \\
& \ldots \\
& \\
&\left.\quad \ldots \quad \|\left(\begin{array}{cc}
1 & \cos \left[\omega_{M}\right] \\
\cos \left[\omega_{M}\right] & \vdots \\
\vdots & \sin \left[\omega_{M}(N-1)\right]
\end{array}\right)\right]
\end{aligned}
$$

where each sinusoid $\omega_{m}=2 \pi m f_{0}$ is represented by a couple of column vectors and the operator $\|$ just signifies column augmentation, that is, removing the abutting parentheses and making a wider matrix out of the operands of the operator $\|$. Using the same notation, the unknown parameter vector $\boldsymbol{\theta}$ can be expressed as $\boldsymbol{\theta}=\left[\left(\alpha_{1} \beta_{1}\right)\|\ldots\|\left(\alpha_{M} \beta_{M}\right)\right]^{T}$, where $\alpha_{m}=A_{m} \cos \phi_{m}$ and $\beta_{m}=-A_{m} \sin \phi_{m}$.

\section{B. Experimental setup}

The detector evaluation has been conducted by studying the performance of the Receiver Operating Characteristic (ROC) curves, where the PD is calculated for every possible PFA. On one hand, this requires the generation of random variables corresponding to non-Gaussian and non-independent noise denoted in the following as $w_{d}$. They are obtained by first generating non-Gaussian independent random variables $w_{i}$ leading to noise vectors $\mathbf{w}_{i}$ and, after that, transforming them as follows $\mathbf{w}_{d}=\mathbf{U}^{-1} \mathbf{w}_{i}$. The elements of the mixture matrix $\mathbf{U}^{-1}$ (dimension $N \times N$ ) are obtained from a random variable with a standard uniform distribution on the open interval $(0,1)$. Therefore, a total number of $3 \cdot 10^{4}$ noise vectors are generated for each simulation. On the other hand, it is also required to generate the subspace signals. In this case, we set the frequency $f_{0}$ to $0.1, \phi_{m}$ are samples of a uniform random variable between $[-\pi-\pi]$ and $A_{m}$ are selected for every required signal to noise ratio (SNR) defined as $S N R=\sum_{m=1}^{M}\left|A_{m}\right|^{2} / \sigma_{\mathbf{w}_{i}}^{2}$.

In order to evaluate the GMSF, we assume that the estimate of the linear transformation $\mathbf{U}$ and the pre-processing function $g($.$) required$ in (6) are obtained in a previous training step where only noise is present. Thereby, selecting the number of noise training vectors $K$, used for the estimation of $\mathbf{U}$, becomes an important problem of practical interest. Obviously, good estimates of $\mathbf{U}$ require a relative great value of $K$. However, it cannot be made arbitrarily large as there are some limiting factors, namely, the computational burden and the time interval duration allowed for training. In general, this decision may be considered part of the overall calibration of the detector for each application, but unfortunately, it is not easy to find an analytic closed equation giving us the best values. Instead, we use an experimental fitting taking the value that results in the best performance in terms of the ROC curves. For example, in Fig.1

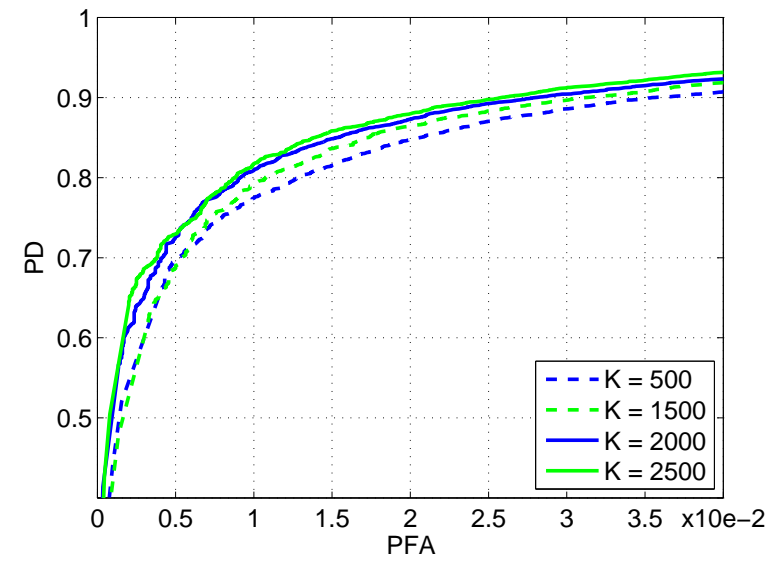

Fig. 1: ROC curves of GMSF for non-Gaussian and non-independent noise generated from Laplacian PDF with $S N R=-2 d B, N=25$, $M=1$ and different $K$ setups.

we show the results obtained for non-Gaussian and non-independent noise $\mathbf{w}_{d}$ (generated from Laplacian PDF) with $S N R=-2 d B$, $N=25, M=1$ and different $K$ setups. It can be observed how for $K>2000$ there is not a significant improvement of the ROC curve. Similar results were achieved varying $S N R, N$ and $M$, therefore, in our experiments $K$ was set to 2000 .

Once we have obtained $\hat{\mathbf{U}}$, it is necessary to estimate the nonparametric function $g($.$) . For that, the estimated linear transformation$ $\hat{\mathbf{U}}$ is applied to the $K$ available noise training vectors $\mathbf{w}_{d}$ as follows: $\hat{\mathbf{u}}=\hat{\mathbf{U}} \mathbf{w}_{d}$. This leads to $K$ linearly preprocessed vectors $\hat{\mathbf{u}}$ and to a total set of $K N$ independent samples of the random variable $u$ which will have similar PDFs to the originals $w_{i}$. However, only a subset of $L=N K / 4$ samples will be randomly selected to estimate $g($. using (16) in an effort to reduce the computational requirements. The parameter $L$ was set considering similar experiments to those conducted to determine the most suitable value of $K$.

\section{Comparing GMSF and MSF}

Several experiments were performed in order to assess the improvements of the GMSF with respect to the MSF varying different parameters involved in the detection problem. First, it is of particular interest to observe how the GMSF behaves in presence of independent non-Gaussian noise $\left(\mathbf{w}_{i}\right)$. In Fig. 2, the ROC curves of both detectors are presented when using independent Laplacian noise for $S N R=-2 d B, N=25$ and $M=1$. It can be observed that both detectors behave similarly as expected. Secondly, in Fig. 3, we show the ROC curves obtained with the MSF and the GMSF considering different types of non-independent noises $\left(\mathbf{w}_{d}\right)$ for $S N R=-6 d B$, $N=25$, and $M=1$. As it was described in the previous sections, these dependent noise samples correspond to linearly transformed independent noises having non-Gaussian PDFs: Rayleigh, Laplacian and Gamma. Furthermore, the ROC curve of the MSF is also represented for the same parameters and non-Gaussian noise PDFs, but with independent samples. Comparison of the three curves for all noise distributions indicates that the MSF detector experiments a considerable deterioration with non-independent noise while the GMSF curves practically coincide with the ones corresponding to the MSF for independent noise. Therefore, it is shown the capability of the GMSF to compensate the degradation of the MSF which becomes a random detector in presence of dependent noise. The above results are of particular importance since it is possible to demonstrate the generalization property of the GMSF, which significantly improves 


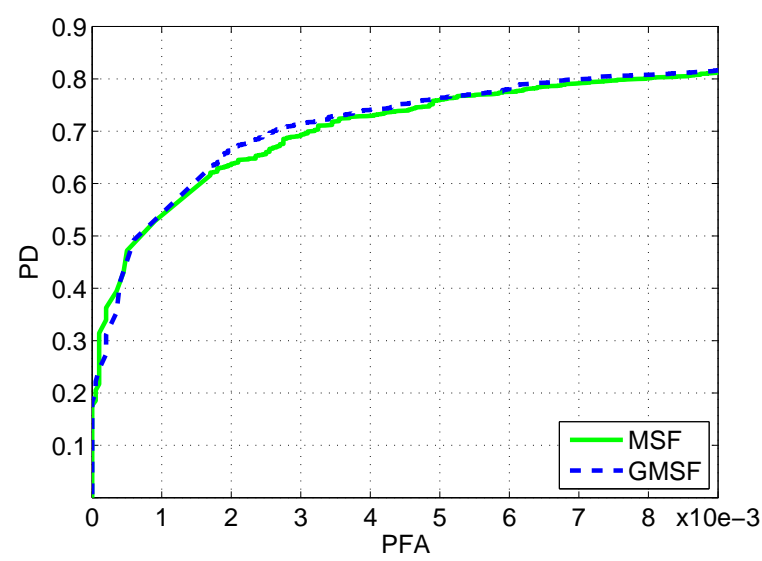

Fig. 2: ROC curves of MSF and GMSF for independent Laplacian noise $\left(\mathbf{w}_{i}\right)$ with $S N R=-2 d B, N=25$ and $M=1$.

the MSF performance in presence of non-independent noise (Fig. 3) and it behaves like the MSF in presence of independent noise (Fig. 2).

In Fig. 4, we extend the previous results testing the influence of varying different parameters involved in the experiments. Fig. 4a, 4b and $4 \mathrm{c}$ show the ROC curves of the GMSF and the MSF for different values of $S N R, N$ and $M$ with non-Gaussian and non-independent noise (generated from Laplacian PDF). In all the examples, the improvements of the GMSF with respect to the MSF are evident and the influence of the parameter value follows the expected behavior. In Fig. 4a we can see how the PD increases with the $S N R$. It must be noticed that the increase is not very significant for the MSF, thus indicating that the $S N R$ should be much higher to compensate the degradation of the MSF in presence of dependent noise. Similarly, in Fig. 4b, as expected, the PD also improves with the observation size $N$ since the normal behavior of any detector implies that the test statistic increases the signal to noise ratio gain with $N$. Again, we can observe how the enhancement in PD when using the MSF is not as significant as the one obtained with the GMSF. Finally, Fig. $4 \mathrm{c}$ shows the influence of varying $M$, the number of involved sinusoids (dimension of the subspace). As expected, the PD increases in the GMSF case when the subspace becomes more restrictive (lower dimension), that is the signal bandwidth becomes narrower, although in MSF this effect is insignificant in comparison with GMSF.

As a general conclusion, it is shown that the degradation of the MSF due to the presence of non-independent noise cannot be easily compensated by increasing the $S N R$, the observation size $N$ or by reducing the signal subspace dimension $M$. Therefore, the use of the GMSF is presented as a practical solution to this problem.

\section{CONCLUSION}

We have introduced a generalization of the matched subspace filters for the detection of unknown signals in a background of non-Gaussian, non-independent noise. To do so the already known detector based on the Rao test, which is applicable to non-Gaussian and independent noise, is modified to include a previous linear transformation based on ICA. In order to obtain general applicability of the proposed test, a non-parametric estimation of the noise PDF is used after the linear transformation. Thereby, the properties of the Rao test are preserved for any kind of non-Gaussian noise distribution. Several experiments were conducted with simulated examples in order to evaluate the MSF in comparison to the GMSF. The study of the resulting ROC curves show the performance enhancement

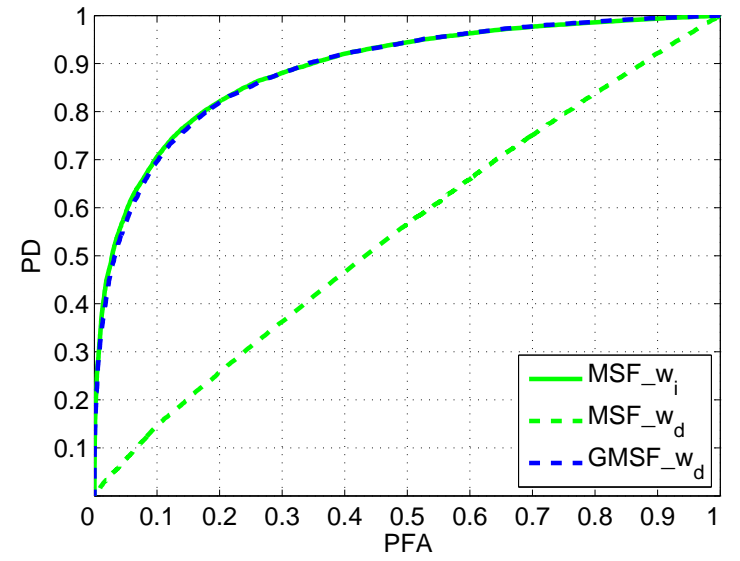

(a)

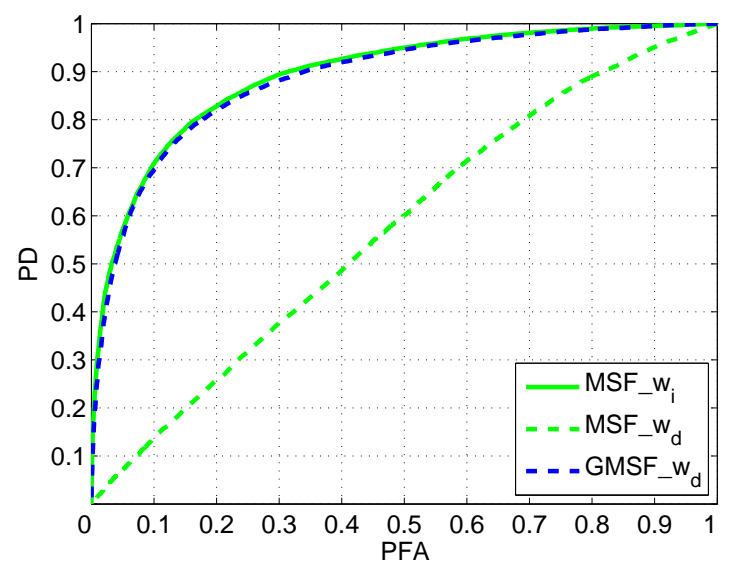

(b)

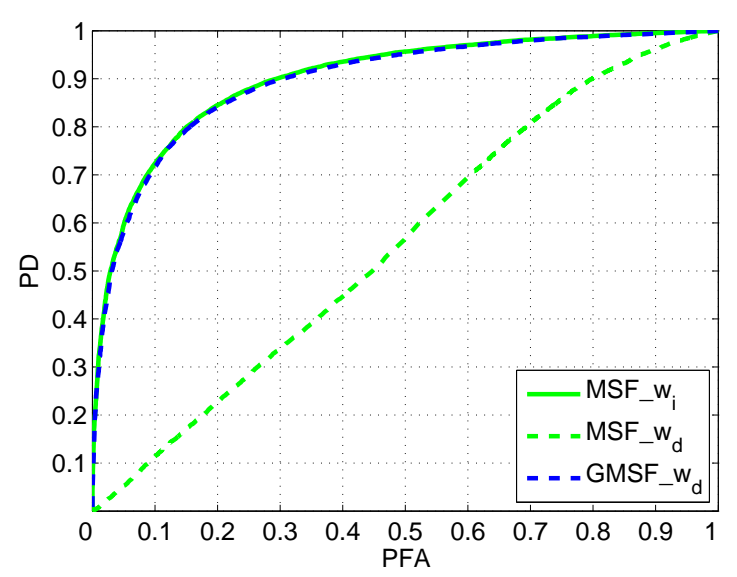

(c)

Fig. 3: ROC curves of MSF and GMSF for different types of nonindependent $\left(\mathbf{w}_{d}\right)$ and independent $\left(\mathbf{w}_{i}\right)$ non-gaussian noise distributions for $S N R=-6 \mathrm{~dB}, N=25$ and $M=1$. (a) Rayleigh noise; (b) Laplacian noise and (c) Gamma noise.

obtained with the generalized solution, and, more specifically that the degradation of the MSF in presence of dependent noise cannot be easily compensated except by using the GMSF. 


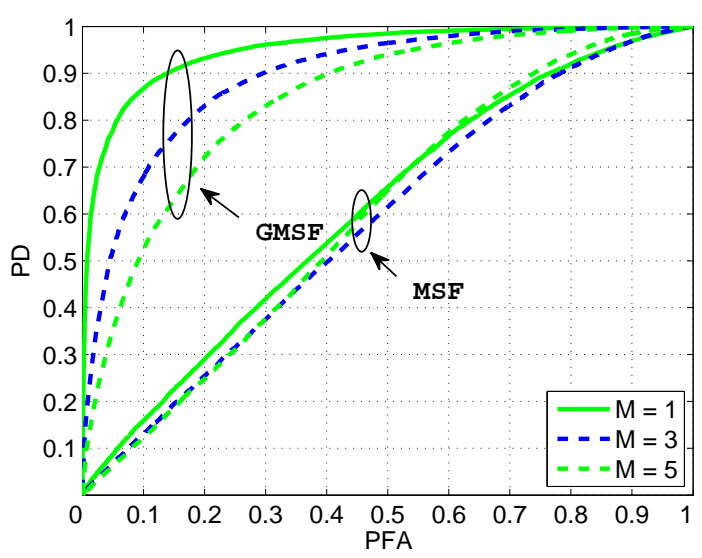

(a)

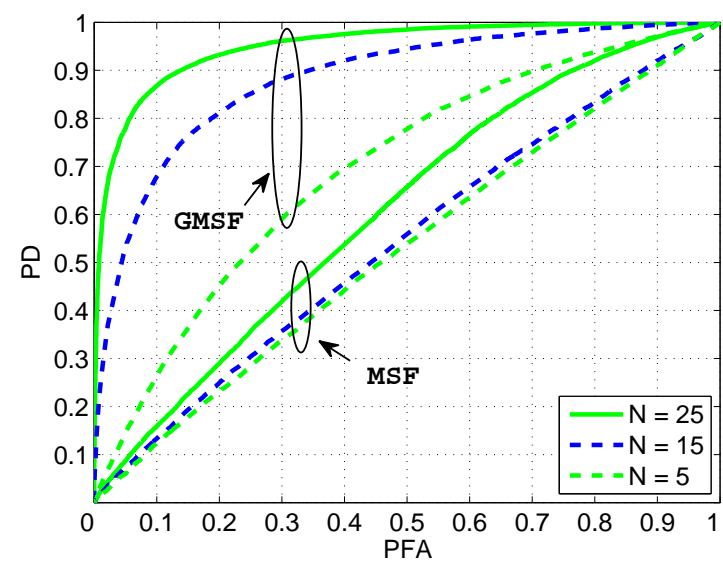

(b)

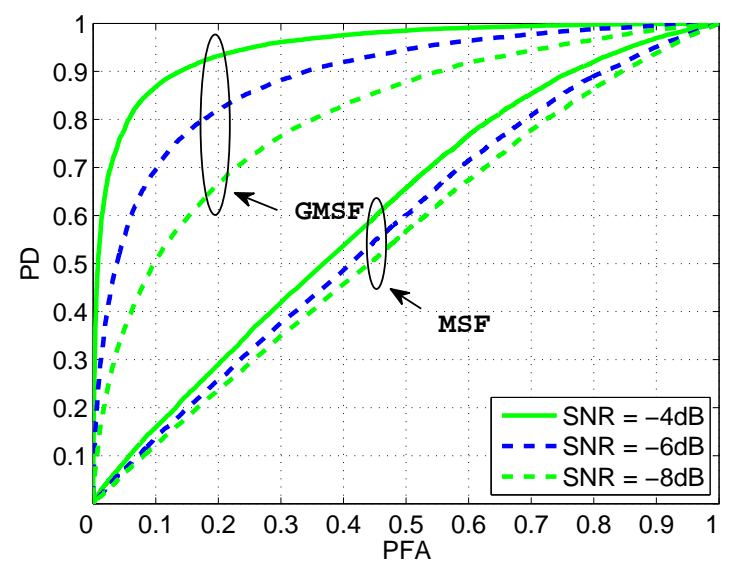

(c)

Fig. 4: ROC curves of MSF and GMSF with non-Gaussian and nonindependent noise distributions generated from Laplacian PDF. (a) $N=25, M=1$ and different $S N R$; (b) $S N R=-4 d B, M=1$ and different observation vector length $(N)$; (c) $S N R=-4 d B$, $N=25$ and different subspace matrix dimension $(M)$.

\section{ACKNOWLEDGMENT}

This work has been supported by Spanish Administration and the FEDER Programme of the European Community under Grant TEC 2008-02975; and by the Generalitat Valenciana under Grant PROMETEO/2010/040.

\section{REFERENCES}

[1] L. L. Scharf, Statistical Signal Processing: detection, estimation, and time series analysis, Addison Wesley, 1991.

[2] L.L. Scharf and B. Friedlander, "Matched subspace detectors," IEEE Transactions on Signal Processing, vol. 42, no. 8, pp. 2146 -2157, 1994.

[3] M.N. Desai and R.S. Mangoubi, "Robust gaussian and non-gaussian matched subspace detection," IEEE Transactions on Signal Processing, vol. 51, no. 12, pp. 3115 - 3127, 2003.

[4] S. M. Kay, Fundamentals of Statistical Signal Processing: Detection Theory, NJ: Prentice-Hall, 1st edition, 1998.

[5] A. De Maio and S. Iommelli, "Coincidence of the rao test, wald test, and glrt in partially homogeneous environment," IEEE Signal Processing Letters, vol. 15, pp. $385-388,2008$.

[6] A. De Maio, S.M. Kay, and A. Farina, "On the invariance, coincidence, and statistical equivalence of the glrt, rao test, and wald test," IEEE Transactions on Signal Processing, vol. 58, no. 4, pp. 1967 -1979, 2010.

[7] Lee T.-W., Independent component analysis: theory and applications, Kluwer Academic Publishers, 1st edition, 1998.

[8] A. Hyvrinen, "Independent component analysis: algorithms and applications," Neural Networks, vol. 13, no. 4-5, pp. 411-430, 2000.

[9] J. Moragues, L. Vergara, J. Gosálbez, and I. Bosch, "An extended energy detector for non-gaussian and non-independent noise," Signal Processing, vol. 89, no. 4, pp. 656, 2009.

[10] D.W. Scott and S.R. Sain, "Multidimensional density estimation," in: C.R. Rao, E.J. Wegman (Eds), Handbook of Statistics, vol. 23: Data Mining and Computational Statistics, August 2004.

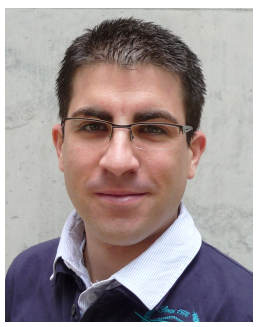

Jorge Moragues was born in Gandia (Spain) in 1981. He received the degree in Telecommunications Engineering in 2006 and the M.S. degree in Technologies, Systems and Communications Networks in 2008, both from the Polytechnic University of Valencia (UPV), Spain. He is a Ph.D. candidate in the Institute of Telecommunications and Multimedia Applications (iTEAM), UPV, and member of the Signal Processing Group (GTS). He stayed at the "Universitt Karlsruhe (TH)" as a researcher within the department of Electrical Engineering and Information Processing and in cooperation with Fraunhofer IOSB. His current research interests are focused on the statistical signal processing area, more specifically related with signal detection, array processing, and decision fusion applied to acoustic signals and to audio-video surveillance systems.

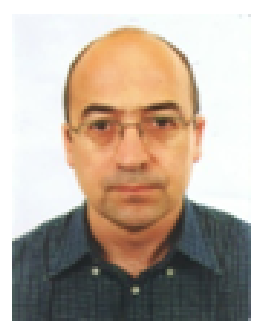

Luis Vergara was born in Madrid (Spain) in 1956. $\mathrm{He}$ received the Ingeniero de Telecomunicacin and the Doctor Ingeniero de Telecomunicacin degrees from the Universidad Politcnica de Madrid (UPM) in 1980 and 1983 respectively. Until 1992 he worked at the Departamento de Señales, Sistemas y Radiocomunicaciones (UPM) as an Associate Professor. In 1992 he joined the Departamento de Comunicaciones (Universidad Politcnica de Valencia UPV, Spain), where he became Professor and where it was Department Head until April 2004. From April 2004 to April 2005 he was Vicerector of New Technologies at the UPV. He is now responsible of the Signal Processing Group of the UPV, a member group of the Institute of Telecommunication and Multimedia Applications (I-TEAM) of UPV. His research concentrates in the statistical signal processing area, where he has worked in different theoretical an applied problems, many of them under contract with the industry. His theoretical aspects of interest are signal detection and classification, independent component analysis and spectral analysis. Currently he is involved in ultrasound signal processing for non-destructive evaluation, in infrared signal processing for fire detection and in cognitive audio for surveillance applications. He has published more than 150 papers including journals and conference contributions. 


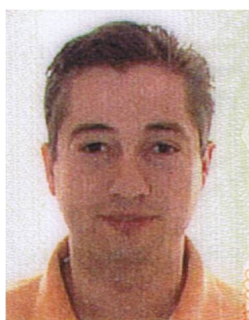

Jorge Gosálbez was born in Valencia (Spain) in 1975. He received Telecomunications Engineering and $\mathrm{PhD}$ degrees from the Universidad Politcnica de Valencia (UPV) in 2000 and 2004 respectively. $\mathrm{He}$ is Associate Professor at Departamento de Comunicaciones (UPV) and member of the Signal Processing Group of the Institute of Telecommunication and Multimedia Applications (I-TEAM) of UPV. His research concentrates in the statistical signal processing area, where he has worked in different theoretical an applied problems, many of them under contract with the industry. His theoretical aspects of interest are time-frequency analysis, signal detection and array processing. Currently he is involved in ultrasound signal processing for non-destructive evaluation of materials, in surveillance systems based on acoustic information and in acoustic source location and tracking based on sensor and array signal processing. He has published more than 50 papers including journals and conference contributions. 